Localizador: 19015 doi: $10.35366 / 93980$

\title{
Carcinoma de células de Merkel. Estudio observacional retrospectivo (1998-2018) del Hospital Universitario Basurto
}

\author{
Merkel cell carcinoma. A retrospective observational \\ study (1998-2018) in a University Hospital
Nerea González-Romero,* Ane Lobato-Izagirre, * Laura Blanch-Rius, ${ }^{\ddagger}$
Goikoane Cancho-Galán, ${ }^{\S}$ Rosa Izu-Belloso ${ }^{\ddagger}$

Palabras clave: Carcinoma de células de Merkel, epidemiología tratamiento.

Keywords:

Merkel cell carcinoma epidemiology, treatment.

* Médico Interno Residente (MIR) de Dermatología.

‡ Dermatóloga.

§ Anatomopatóloga.

Servicio de Dermatología, Hospital Universitario

Basurto. Bilbao, España.

Conflicto de intereses:

Ninguno.

Recibido:

30/Marzo/2019.

Aceptado:

30/Julio/2019.

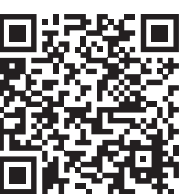

\section{RESUMEN}

Introducción: El carcinoma de células de Merkel (CCM) es una neoplasia cutánea infrecuente y de pronóstico pobre. No hay consenso para abordar su manejo. Material y métodos: Realizamos una búsqueda de pacientes con diagnóstico histológico de CCM entre 1998 y $2018(n=20)$, en la base de datos de nuestro hospital. Recogimos las siguientes variables: sexo, edad, historia de inmunosupresión y de cáncer cutáneo no melanoma y melanoma maligno, sospecha diagnóstica inicial, tamaño tumoral, localización, estadificación, tratamientos recibidos, evolución y supervivencia. Resultados: La mayoría de las lesiones (40\%) se localizaban en la cabeza y cuello, y 50\% tenían un tamaño de 2 a $5 \mathrm{~cm}$. Ochenta y cinco por ciento de los pacientes recibió tratamiento quirúrgico y $75 \%$ radioterapia. Cincuenta por ciento de los pacientes presentaron recidivas, todas en los primeros dos años. Hemos observado que la supervivencia es mayor en los pacientes con lesiones menores de $2 \mathrm{~cm}$, localizadas en extremidades, con positividad para poliomavirus, en los que recibieron radioterapia adyuvante y no presentan márgenes quirúrgicos afectados. Conclusiones: Hemos identificado cinco factores asociados con una mayor supervivencia que, aunque no son estadísticamente significativos (por el tamaño muestral), son clínicamente relevantes y coinciden con los de otras series.

\section{ABStRACT}

Introduction: Merkel cell carcinoma (MCC) is a rare cutaneous neoplasm with a poor prognosis. There is no consensus to address its management. Material and methods: We performed a search of patients with histological diagnosis of MCC from 1998 to $2018(n=20)$, in the database of our hospital and we collected next data: sex, age, history of immunosuppression, skin cancer no melanoma and malignant melanoma, initial diagnosis suspicion, tumor size, location, staging, treatments received, evolution and survival. Results: The majority of lesions (40\%) were located in the face and neck and $50 \%$ had 2 to $5 \mathrm{~cm}$. $85 \%$ of the patients received surgical treatment, $75 \%$ had RT. $50 \%$ of patients had recurrence, all before the first 24 months. In our sample, we observed that survival is greater in patients with lesions smaller than $2 \mathrm{~cm}$, located in extremities, positive for polyomavirus, in those who have received adjuvant $R T$ and in those without affected surgical. Conclusions: We have observed five factors associated with grater survival, which, although not statistically significant, are clinically relevant and concur with those of other series.

\section{INTRODUCCIÓN}

$\mathrm{E}$ I carcinoma de células de Merkel (CCM) es 4 una neoplasia cutánea muy poco frecuente que predomina en varones de edad avanzada. ${ }^{1}$ Se manifiesta como un nódulo o tumoración firme rojo-violácea, habitualmente de 2 a 4 $\mathrm{cm},{ }^{1}$ localizado de manera frecuente en áreas fotoexpuestas, ${ }^{1}$ siendo la cabeza y el cuello $(\mathrm{CyC})$ la localización más habitual $(50 \%){ }^{1}$

La exposición a la radiación ultravioleta (UV), la inmunosupresión y la presencia del poliomavirus en el genoma del tumor (presente en $80 \%$ de los casos) son los tres factores más importantes asociados con su etiopatogenia. ${ }^{1,2}$

En general, la sospecha clínica inicial no suele ser de CCM ${ }^{1}$ confundiéndose de modo usual con otras neoplasias malignas como el carcinoma espinocelular. ${ }^{1}$

El pronóstico es pobre, y no es infrecuente que haya afectación locorregional al realizar el diagnóstico, ${ }^{3}$ e incluso que la enfermedad esté diseminada. ${ }^{1}$ Por ello, se debe solicitar un estudio de extensión al diagnóstico. ${ }^{3} \mathrm{Si}$ 
la enfermedad está localizada la mayoría de expertos recomiendan realizar biopsia de ganglio centinela (BGC) en todos los casos. ${ }^{3,4}$ De hecho, esta recomendación está incluida en las guías de la National Comprehensive Cancer Network (NCCN), ${ }^{3,4}$ ya que es un indicador pronóstico que permite identificar mejor a los pacientes que se benefician de una terapia postquirúrgica regional ${ }_{1}^{4}$ incrementado así su supervivencia. ${ }^{5}$ En aquellos pacientes con BGC positiva se aconseja realizar linfadenectomía y/o radioterapia (RT) sobre el lecho ganglionar. ${ }^{3,4}$

No hay un consenso para abordar el manejo del CCM. Sin embargo, parece claro que el tratamiento principal (en los estadios I-III) es quirúrgico. ${ }^{3,4}$ Tampoco se ha establecido un margen quirúrgico óptimo. ${ }^{4}$ La guía de la NCCN recomienda realizar ampliación de márgenes de 1 a $2 \mathrm{~cm}^{3,4}$ seguido de RT sobre el lecho tumoral, aunque se ha observado una disminución de las recurrencias al ampliar a $3 \mathrm{~cm}$, sin que se haya demostrado un incremento de la supervivencia global ${ }^{3}$ y, aun así, la tasa de recurrencias oscila entre 25 y $40 \% .{ }^{4}$ Por ello, habrá que valorar en cada caso la morbilidad quirúrgica a la hora de optar por un margen mayor o menor. ${ }^{4}$

La RT parece disminuir también las recidivas locales, ${ }^{3} \mathrm{e}$ incluso aumenta la supervivencia global en los pacientes con enfermedad localizada (El y EII). ${ }^{4}$ Por eso, al seguir las recomendaciones de la $\mathrm{NCCN}$, algunos centros indican la RT sobre el lecho quirúrgico en los pacientes con BGC negativa, incluyendo también la región ganglionar si la BGC fuera positiva o en los casos en los que no se hubiera podido realizar. ${ }^{4}$ Sin embargo, otros prefieren no emplear ésta de forma rutinaria. ${ }^{3}$

Por último, desde 2017, el avelumab (anticuerpo monoclonal anti PD-L1) es el tratamiento de primera línea en pacientes con CCM metastásico. ${ }^{4,6}$

\section{MATERIAL Y MÉTODOS}

Estudio observacional, descriptivo y retrospectivo de los pacientes diagnosticados histológicamente con CCM en un hospital terciario durante 20 años (1998-2018). Se empleó la base de datos del Servicio de Anatomía Patológica para realizar la búsqueda. Se obtuvo el permiso del Comité Ético de Investigación Clínica de nuestro hospital.

Se recogieron de la historia clínica las características epidemiológicas (sexo, edad al diagnóstico), antecedentes personales relacionados con la inmunosupresión (neoplasia sólida, neoplasia hematológica, VIH, trasplante, fármacos inmunosupresores IS), historia de cáncer cutáneo no melanoma (CCNM) y de melanoma maligno (MM), sospecha inicial de CCM u otra neoplasia maligna, tamaño del tumor $(5 \mathrm{~cm})$, localización del tumor $(\mathrm{CyC}$, tronco, extremidades superiores o extremidades inferiores), estadificación inicial (I, II, III o IV, según la clasificación de la American Joint Committee on Cancer, AJCC), tratamientos recibidos tanto en el diagnóstico inicial como en la recidiva locorregional o en la progresión a distancia (cirugía, RT, quimioterapia QT y/o avelumab), ampliación de márgenes quirúrgicos $(0,0.5,1,1.5,2,2.5,3 \mathrm{o}>3 \mathrm{~cm})$, realización o no de BGC y/o de linfadenectomía, desarrollo de recidiva local y/o regional o progresión a distancia a lo largo del seguimiento y supervivencia (meses).

Para realizar un análisis descriptivo de nuestra serie calculamos las frecuencias absolutas y relativas de las variables cualitativas y la media, la desviación estándar, la mediana y el rango de las variables cuantitativas. La asociación entre las distintas variables con la supervivencia se calculó mediante el análisis de la varianza (ANOVA) en caso de variables cualitativas (sexo, poliomavirus, localización tumoral, tamaño tumoral, márgenes quirúrgicos afectados, RT asociada, QT asociada, avelumab asociado) y el coeficiente de correlación de Spearman en caso de variable cuantitativa (edad). La asociación entre la aparición de recidivas (locorregionales o a distancia) con la afectación de márgenes quirúrgicos y uso de RT o QT se analizó mediante el test de $\chi^{2}$. El nivel de significación estadística se fijó en 0.05 .

El objetivo del estudio es conocer las características epidemiológicas, clínicas y el manejo diagnóstico y terapéutico de los pacientes con CCM en nuestra población

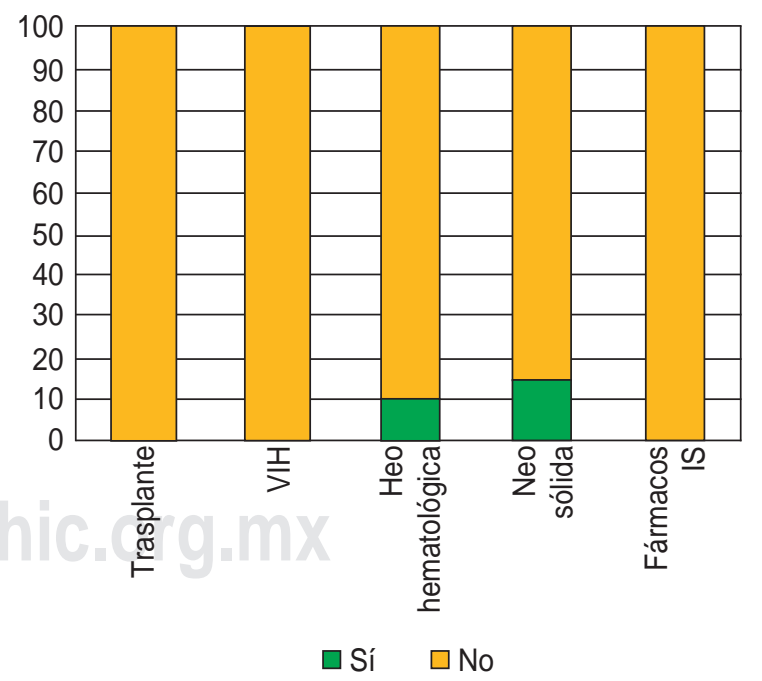

Figura 1: Comorbilidades asociadas con inmunosupresión. Dos pacientes presentaban antecedentes de inmunosupresión y en ambos casos la causa era una neoplasia hematológica. 


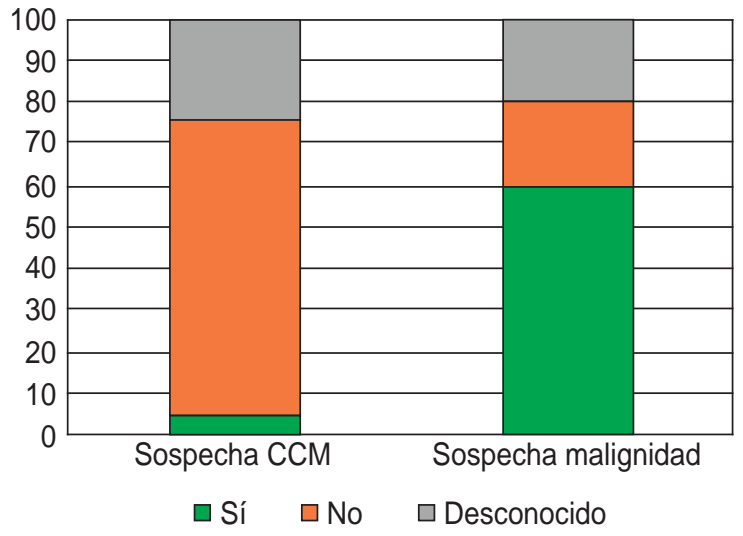

Figura 2: Sospecha diagnóstica inicial. Aunque la sospecha más frecuente (60\%) fue de malignidad, sólo se sospechó CCM en un caso. En los cuatro casos en los que se pensó en tumoración benigna, la sospecha fue de un médico no dermatólogo.

Tabla 1: Sospecha diagnóstica inicial de lesión maligna. La más frecuente fue carcinoma espinocelular.

\begin{tabular}{|ll|}
\hline Sospecha diagnóstica inicial & n (\%) \\
\hline Carcinoma espinocelular & $3(15)$ \\
Carcinoma basocelular & $2(10)$ \\
Linfoma cutáneo & $2(10)$ \\
MM amelanótico & $2(10)$ \\
Metástasis cutánea & $1(5)$ \\
Angiosarcoma & $1(5)$ \\
Acantoma de células claras & $1(5)$ \\
\hline MM = Melanoma maligno. & \\
\hline
\end{tabular}

de referencia de los últimos 20 años, así como conocer los factores pronósticos en relación con la supervivencia.

\section{RESULTADOS}

En total estudiamos 20 pacientes, 11 mujeres (55\%) y nueve hombres (45\%). La media de edad al diagnóstico fue de 76.55 años con una mediana de 79 años. Diez por ciento tenían historia de neoplasia hematológica y $15 \%$ de neoplasia sólida. Ninguno con historia de neoplasia sólida cumplía criterios de inmunosupresión (Figura 1). Ocho (40\%) habían presentado algún CCNM y sólo uno (5\%) MM.

Al inicio sólo se sospechó CCM en un caso (5\%) (Figura 2). La sospecha inicial fue de neoplasia maligna (Tabla 1) en 12 casos $(60 \%)$ y en los seis casos $(20 \%)$ en los que la sospecha inicial no fue de lesión maligna (Tabla 2), el diagnóstico lo hizo un médico que no era dermatólogo.
Cuarenta por ciento de las lesiones se localizaban en la CyC, seguido de las extremidades superiores en 35\% (Figura 3). Cincuenta por ciento tenían un tamaño entre 2 y $5 \mathrm{~cm}$ (Figura 4). En los tumores de $2 \mathrm{~cm}$ la supervivencia fue de 112 meses, disminuyendo a la mitad en aquellos mayores de $2 \mathrm{~cm}$.

En 12 pacientes se llevó a cabo la detección del poliomavirus de las células de Merkel (MCPyV) y fue positiva en siete de ellos (58.3\%).

Cuarenta y cinco por ciento se encontraban en un estadio precoz de la enfermedad ( $35 \%$ en estadio II y $10 \%$ en I). Sin embargo, $50 \%$ presentaba enfermedad regional (estadio III) y un caso (5\%) presentaba enfermedad diseminada a distancia (estadio IV) en el momento del diagnóstico.

Ochenta y cinco por ciento recibió tratamiento quirúrgico (Figura 5); sin embargo, no queda claro el criterio empleado para la elección de los márgenes de ampliación quirúrgicos. En 25\% de los casos se optó por una ampliación de $2 \mathrm{~cm}$, en $15 \%$ de $2.5 \mathrm{~cm}$, en dos casos (10\%) se realizó una ampliación de $3 \mathrm{~cm}$, en otros dos $(10 \%)$ de $1.5 \mathrm{~cm}$, en uno se hizo una ampliación de $0.5 \mathrm{~cm}$ y en otro se efectuó una resección paliativa sin ampliación de márgenes. Hubo dos casos en los que se realizó amputación de un dedo de la mano (Figura 6).

\section{Tabla 2: Sospecha diagnóstica inicial de lesión benigna.}

\begin{tabular}{ll}
\hline Sospecha diagnóstica inicial & $\mathrm{n}(\%)$ \\
\hline Granuloma piogénico & $1(5)$ \\
Absceso & $1(5)$ \\
Tofo gotoso & $1(5)$ \\
Hernia & $1(5)$ \\
Quiste epidérmico & $1(5)$ \\
Quiste triquilemal & $1(5)$ \\
\hline
\end{tabular}

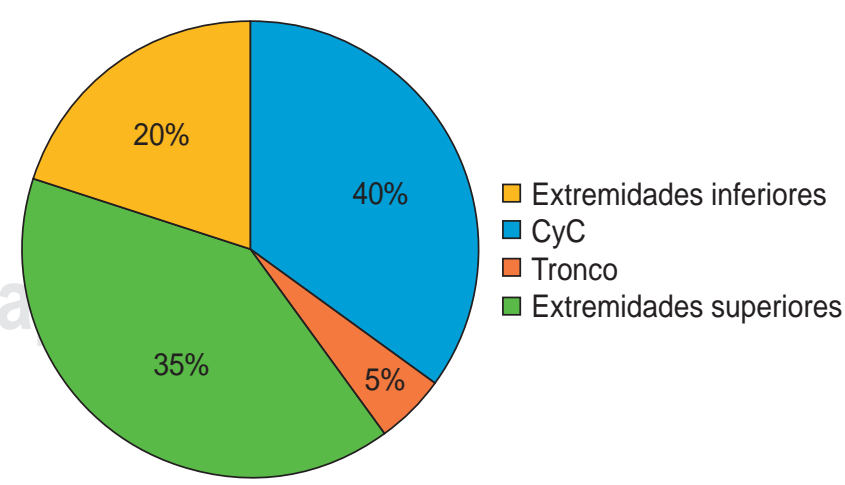

Figura 3: Distribución de la muestra en función de localización del tumor. La localización más frecuente fue cabeza y cuello (40\%). 


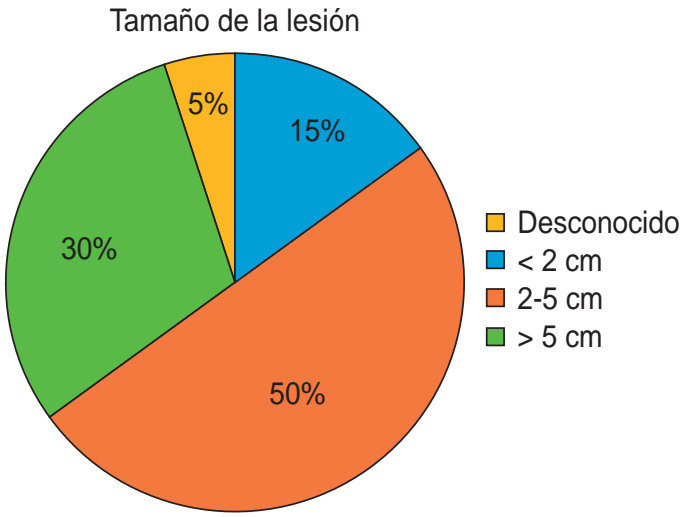

Figura 4: Distribución de la muestra en función del tamaño del tumor. La mayoría (80\%), mayor de $2 \mathrm{~cm}$.

La BGC no se realizó de forma sistemática; de hecho, sólo se realizó en tres pacientes (EI/EII), por lo que no lo hemos podido incluir en el análisis de los datos ni hemos podido obtener ninguna conclusión al respecto. En los tres casos, el resultado fue negativo.

Quince pacientes (75\%) recibieron RT: en 13 (65\%) fue empleada como terapia adyuvante a la cirugía (de éstos, $38.46 \%$ eran estadios precoces, el resto eran EIII) y en cinco (25\%) fue empleada como tratamiento de recidivas locorregionales. Sólo en cinco casos tratados con cirugía no se empleó RT, tres de ellos eran estadios localizados (uno El, dos Ell y dos EIII). No queda reflejado en la historia clínica el motivo por el que se tomó esa decisión.

En cuatro casos se empleó QT, en tres de ellos por recurrencia locorregional o progresión a distancia de la enfermedad. En dos de ellos se administró además avelumab: en un caso por recidiva locorregional y en el otro por progresión a distancia de la enfermedad. En estos pacientes, la media de supervivencia fue menor que los que no recibieron estos tratamientos (22.25 meses vs 62.06 meses, $p=0.304$, IC95\% [-39.18, 118.81]). Cincuenta por ciento de los pacientes presentó algún tipo de recidiva (local y/o regional), 45\% de los pacientes no presentaron recidiva y en el $5 \%$ restante se desconoce este dato. En cinco pacientes (25\%) se observó progresión a distancia de la enfermedad.

Sesenta y dos punto cinco por ciento de los pacientes que presentaron recidiva local ( $p=0.28$, IC $95 \%$ $[-0.78,0.21])$ y todos los pacientes que presentaron progresión a distancia tenían los márgenes quirúrgicos afectados ( $p=0.051$ IC95\% [-0.79, -0.06]). Estos resultados son clínicamente relevantes; sin embargo, no son estadísticamente significativos, dado que la muestra es pequeña. Todas las recidivas se produjeron en los primeros 24 meses.

La media de supervivencia de nuestra muestra fue de 54 meses (4.5 años) y la mediana de 22 meses. La supervivencia a los 12 meses fue de 75\%. De éstos, 53.3\% eran estadios precoces (EI o EII); $26.6 \%$ EIII y $0.066 \%$ EIV. La supervivencia a los 24 meses fue de 45\%. De ellos, 66.6\% eran EI o EII, 33.3\% eran EIII y ninguno EIV. Ochenta y ocho punto ocho por ciento de los estadios precoces sobrevivieron 12 meses y $66.6 \% 24$ meses, mientras que sólo $60 \%$ de los Elll sobrevivieron 12 meses y sólo 30\% 24 meses.

Los factores que en nuestra serie se relacionan con una mayor supervivencia son los siguientes: el tamaño tumoral menor de $2 \mathrm{~cm},(p=0.307$, IC95\% [-55.90, 181.57]), la ausencia de afectación de márgenes quirúrgicos ( $\mathrm{p}=$ 0.051, IC95\% [-0.36, 140.86]), la localización en EESS y EEII ( $p=0.179)$, la utilización de RT adyuvante $(\mathrm{p}=$ 0.335, IC95\% [-107.77, 38.71]) y la positividad para el poliomavirus ( $p=0.194$, IC95\% [-160.28, 37.08]). En todos ellos se observan diferencias clínicamente relevantes sin llegar a la significación estadística.

\section{DISCUSIÓN}

El CCM representa un pequeño porcentaje dentro de las neoplasias malignas cutáneas, por lo que no existen algoritmos diagnósticos y terapéuticos consensuados basados en una evidencia científica sólida, ya que las series publicadas en la literatura suelen presentar un tamaño muestral pequeño.

Es más habitual en varones de edad avanzada. Sin embargo, hay varias series de casos que, como en la nuestra, el

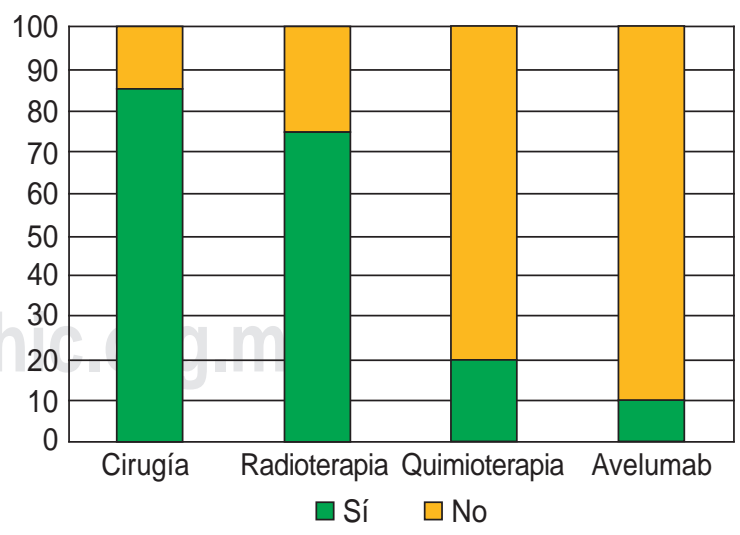

Figura 5: Tratamientos recibidos. La mayoría de los pacientes fueron tratados con cirugía y $R T$. 
predominio es en mujeres. ${ }^{7,8}$ En la mayoría de las series, ${ }^{7-9}$ al igual que en la nuestra, la edad media de diagnóstico es de 70-80 años y la localización más frecuente es en CyC seguida de las extremidades.

Los tumores localizados en glúteos, extremidades inferiores o tronco se han relacionado con una menor supervivencia; ${ }^{8}$ sin embargo, algunos autores ${ }^{9}$ han encontrado que aquellos casos localizados en las extremidades superiores tienen una mayor supervivencia. Los resultados de nuestro estudio coinciden con esto último.

La exposición a radiación UV es el factor de riesgo más relevante en la etiopatogenia de esta neoplasia. De hecho, en nuestra serie, $40 \%$ de los pacientes habían presentado algún CCNM, una proporción mayor que en otras series como la de Dañino-García y colaboradores ${ }^{9}$ en la que $26.32 \%$ presentaban antecedentes de CCNM o como en la de Llombart y colegas ${ }^{8}$ que fue de $30 \%$. Probablemente, esta menor proporción de casos se deba a que el CCNM no es un antecedente que se suela recoger en la historia clínica.

Otro factor relacionado con el desarrollo de CCM es la inmunosupresión. En nuestra serie, 10\% presenta algún tipo de inmunosupresión, dato similar a los publicados en otras. ${ }^{9,10}$ Asimismo, en la nuestra, como en la mayoría de las series de la literatura, ${ }^{9}$ la sospecha inicial más frecuente fue carcinoma espinocelular. Sólo en 5\% de los casos se sospechó CCM.

El tamaño medio del tumor primario en nuestro estudio es de 2 a $5 \mathrm{~cm}$ coincidiendo con lo descrito en la literatura. ${ }^{8,9}$ En cambio, hay otras ${ }^{7,10}$ que presentan un tamaño medio inferior a $2 \mathrm{~cm}$, lo que pone de manifiesto una mayor capacidad de detección precoz del CCM.

En 58.3\% de los pacientes se encontró positividad para poliomavirus, una proporción algo inferior a la descrita en la literatura $(80 \%){ }^{2}$

Muchos autores ${ }^{1,9}$ han encontrado que un tamaño tumoral mayor de $2 \mathrm{~cm}$ es un factor de mal pronóstico. Nuestra serie coincide con esto, ya que aquéllos con tumores menores de $2 \mathrm{~cm}$ presentaron mayor supervivencia.

Aunque el tamaño del tumor primario es un factor pronóstico importante, su relación con la afectación ganglionar es controvertida. Stokes y su equipo indican que la BGC no está justificada en tumores menores de $1 \mathrm{~cm}$. Sin embargo, otros autores como Fields y colegas y Schwartz y su grupo observaron que los tumores de ese tamaño tienen un riesgo de $24-26 \%$ de presentar enfermedad ganglionar. Un estudio retrospectivo ${ }^{10}$ corrobora esto último, al observar que los sujetos con tumores primarios pequeños tienen un riesgo significativo de presentar enfermedad ganglionar al diagnóstico y concluyen que no hay un tamaño para el tumor primario en el cual el riesgo de afectación ganglionar sea despreciable y por lo tanto, consideran que no debería emplearse el tamaño tumoral para descartar el estudio ganglionar en un paciente. La revisión realizada por Tello y colaboradores ${ }^{4}$ coincide con este criterio. Como se comentó en los resultados de nuestra serie, la BGC sólo se realizó en tres casos, en consecuencia no podemos obtener ninguna conclusión al respecto. En el futuro, deberá plantearse realizarla en todos los casos de enfermedad localizada, tal y como recomiendan las guías y la mayoría de los expertos.

En cuanto a la estadificación al diagnóstico, en nuestro estudio tenemos una mayor proporción de casos con ElII (50\%) y una menor proporción de estadios precoces (45\%) que en otras series de la literatura. ${ }^{8,9}$ Estos resultados, de nuevo, revelan un diagnóstico tardío por la dificultad en la sospecha diagnóstica.

La cirugía es el pilar del tratamiento de esta neoplasia. De hecho, 85\% de nuestros pacientes recibieron tratamiento quirúrgico. En un número elevado de casos de nuestra serie se realizó una ampliación mayor de $2 \mathrm{~cm}$ (25\%), superior a lo recomendado por la NCCN. ${ }^{4} \mathrm{~A}$ pesar de que hay estudios ${ }^{11}$ que han sugerido que márgenes de 2 a $3 \mathrm{~cm}$ se asocian con menores tasas de recurrencia local, coincidiendo con otras series, ${ }^{12}$ no obtuvimos diferencias estadísticamente significativas ni clínicamente relevantes en la supervivencia entre aquéllos a los que se les realizó una ampliación de 1-2 cm, frente a los que se les realizó una ampliación mayor.

La RT es el tratamiento complementario empleado en los casos de afectación locorregional. En estadios precoces su empleo es controvertido, aunque cada vez hay más estudios que arrojan mayor evidencia sobre el papel que tiene en el incremento de la supervivencia. ${ }^{13}$ En nuestra serie se empleó RT en 75\% de los pacientes. De los sujetos tratados con RT en un primer tiempo, sólo $38.46 \%$ eran estadios precoces. Deberíamos considerar en el futuro ofrecer en más casos la RT como terapia adyuvante en El y Ell.

Cincuenta por ciento presentó recidivas locorregionales, una proporción similar a la observada en la serie de Llombart y colaboradores ${ }^{8}(45 \%)$, y $25 \%$ progresión a enfermedad a distancia. Todas las recidivas ocurrieron en los primeros dos años, lo cual coincide con lo mencionado en la literatura. ${ }^{4}$ La mayoría con recidiva local y todos los que presentaron enfermedad diseminada tenían márgenes quirúrgicos afectados. Por lo tanto, aunque estas asociaciones no obtuvieron significación estadística, parece relevante que la afectación de márgenes tras la cirugía aumente la 
tasa de recidivas locales y por consiguiente, pueda influir también en el riesgo de diseminación a distancia.

Por último, la supervivencia a los 12 meses en nuestra serie $(75 \%)$ es similar a otras como la de Dañino-García ${ }^{9}$ (78\%); sin embargo, a los 24 meses es inferior (45\% versus $69.3 \%$ ). Esto podría ser debido a que el porcentaje de sujetos con Elll en el momento del diagnóstico en nuestra muestra es superior (50\% versus $21.05 \%$ ).

En nuestra serie, los principales factores relacionados con una mayor supervivencia han sido: el tamaño tumoral menor de $2 \mathrm{~cm}$, la ausencia de afectación de márgenes quirúrgicos, la localización en extremidades superiores o extremidades inferiores, el empleo de RT adyuvante y la positividad para poliomavirus. Estos coinciden en su mayoría con los principales factores de buen pronóstico descritos en la literatura: ${ }^{9}$ localización en miembros superiores, sexo femenino, tumor primario nodal sin lesión cutánea, tumores menores de $2 \mathrm{~cm}$, presencia de poliomavirus y ausencia de lesión ganglionar y metastásica, así como ausencia de inmunosupresión, entre otros.

\section{CONCLUSIONES}

Presentamos una serie de casos de CCM que, para la mayoría de variables, coincide con otras series publicadas. Hemos identificado cinco factores relacionados con una mayor supervivencia, que son clínicamente significativos, aunque dado el pequeño tamaño muestral no alcanzan la significación estadística. En el futuro, debemos plantearnos ofrecer RT adyuvante a la cirugía así como BGC a la mayoría de pacientes El/EII dada su influencia en el pronóstico.

\author{
Correspondencia: \\ Nerea González-Romero \\ Avenida de Montevideo Núm. 18, \\ Pabellón Arrupe $3^{\circ}$ planta (S. Dermatología), \\ 48013, Bilbao (Bizkaia), España. \\ Teléfono: 0034944006063 \\ Fax: 0034944006177 \\ E-mail: nerea.gonzalezromero@osakidetza.eus
}

\section{BIBLIOGRAFÍA}

1. Llombart B, Requena C, Cruz J. Update on Merkel cell carcinoma: epidemiology, etiopathogenesis, clinical features, diagnosis, and staging. Actas Dermosifiliogr. 2017; 108 (2): 108-119.

2. Santos-Juanes J, Fernández-Vega I, Fuentes N, Galache C, Coto-Segura P, Vivanco B et al. Merkel cell carcinoma and Merkel cell polyomavirus: a systematic review and metaanalysis. Br J Dermatol. 2015; 173 (1): 42-49.

3. Llombart B, Kindem S, Chust M. Merkel cell carcinoma: an update of key imaging techniques, prognostic factors, treatment, and follow-up. Actas Dermosifiliogr. 2017; 108 (2): 98-107.

4. Tello TL, Coggshall K, Yom SS, Yu SS. Merkel cell carcinoma: An update and review: Current and future therapy. J Am Acad Dermatol. 2018; 78 (3): 445-454.

5. Gunaratne DA, Howle JR, Veness MJ. Sentinel lymph node biopsy in Merkel cell carcinoma: a 15-year institutional experience and statistical analysis of 721 reported cases. $\mathrm{Br} \mathrm{J}$ Dermatol. 2016; 174 (2): 273-281.

6. Bommareddy PK, Kaufman HL. Avelumab and other recent advances in Merkel cell carcinoma. Future Oncol. 2017; 13 (30): 2771-2783.

7. Ruiz R, Blasco J, Merino J, Linares J, Naranjo R. Carcinoma de células de Merkel. Presentación de 6 casos. Actas Dermosifiliogr. 2003; 94: 300-304.
8. Llombart B, Monteagudo C, López-Guerrero JA, Carda C, Jorda E, Sanmartín O et al. Clinicopathological and immunohistochemical analysis of 20 cases of Merkel cell carcinoma in search of prognostic markers. Histopathology. 2005; 46 (6): 622-634.

9. Dañino-García M, Domínguez-Cruz JJ, Pérez-Ruiz C, ConejoMir J, Pereyra-Rodríguez JJ. Clinical and epidemiological characteristics of Merkel cell carcinoma in a series of 38 patients. Actas Dermosifiliogr. 2019; 110 (5): 360-365.

10. Iyer JG, Storer BE, Paulson KG, Lemos B, Philips JL, Bichakjian $\mathrm{CK}$ et al. Relationships among primary tumor size, number of involved nodes, and survival for 8044 cases of Merkel cell carcinoma. J Am Acad Dermatol. 2014; 70 (4): 637-643.

11. Gollard R, Weber R, Kosty MP, Greenway HT, Massullo V, Humberson C. Merkel cell carcinoma: review of 22 cases with surgical, pathologic, and therapeutic considerations. Cancer. 2000; 88: 1842-1851.

12. Allen PJ, Bowne WB, Jaques DP, Brennan MF, Busam K, Coit DG. Merkel cell carcinoma: prognosis and treatment of patients from a single institution. J Clin Oncol. 2005; 23: 2300-2309.

13. Bhatia S, Storer BE, Iyer JG, Moshiri A, Parvathaneni U, Byrd D et al. Adjuvant radiation therapy and chemotherapy in Merkel cell carcinoma: survival analyses of 6908 cases from the National Cancer Data Base. J Natl Cancer Inst. 2016; 108 (9). pii: djw042. 